\title{
Preparation and Characterization of Conductive Cellulosic Fabric by Polymerization of Pyrrole
}

\author{
Jakup Wiener ${ }^{1}$, Mohamed A. Ramadan ${ }^{1,2}$, Rehab Gomaa ${ }^{3}$, Rehan Abbassi ${ }^{4}$, Ali Hebeish ${ }^{2}$ \\ ${ }^{1}$ Department of Textile Chemistry, Technical University, Liberec, Czech Republic; ${ }^{2}$ Textile Research Division, National Research \\ Center, Dokki, Egypt; ${ }^{3}$ Specific Education Faculty, Zagazig University, Sharkeya, Egypt; ${ }^{4}$ Department of Textile Materials, Techni- \\ cal University, Liberec, Czech Republic. \\ Email: amaramadan1@hotmail.com
}

Received August $25^{\text {th }}, 2013$; revised September $26^{\text {th }}, 2013$; accepted October $7^{\text {th }}, 2013$

Copyright (C) 2013 Jakup Wiener et al. This is an open access article distributed under the Creative Commons Attribution License, which permits unrestricted use, distribution, and reproduction in any medium, provided the original work is properly cited.

\begin{abstract}
Preparation of conductive cotton fabrics, by polymerization of pyrrole in presence of $\mathrm{FeCl}_{3}$ as an oxidizing agent and tetraethylammonium p-toluene sulfonat (TEAp-TS) as a doping agent which in turn caused a reduction in the moisture regain of the substrate, resulted in production conductive cotton fabrics in laboratory scale. Factors affecting the properties of the fabrics were studied, such as ratio between $\mathrm{FeCl}_{3}$ and TEAp-TS and pyrrole concentration. Polypyrrole coated cotton fabrics were characterized by scanning electron microscopy (SEM) and Fourier transform-infrared (FT-IR) spectroscopy. Resistivity, weight gain, color strength, tensile strength and elongation of the coated fabric with polypyrrol were monitored according to ASTM procedures. The results had shown that, the conductivity, weight gain, color strength, tensile strength and elongation increase by increasing $\mathrm{FeCl}_{3}$, TEAp-TS and pyrrole concentration. This is due to the increase of amount of the conductive polymer on the fabric surface. $\mathrm{FeCl}_{3}(0.25 \mathrm{M} / \mathrm{L})$, TEAp-TS $(0.125 \mathrm{M} / \mathrm{L})$, pyrrole $(0.3 \mathrm{M} / \mathrm{L})$, temperature $\left(25^{\circ} \mathrm{C}\right)$ and time $(4 \mathrm{~h})$ represented the most appropriate formulation for effecting polymerizing of pyrrole and anchoring on cotton fabrics.
\end{abstract}

Keywords: Polymerization; Polymer; Pyrrole; Conductivity; Cellulose

\section{Introduction}

Electronic textiles are fabrics/garments that contain electronic circuits, optical fibers or sensors. Such functional textiles provide potential opportunities for boundless applications in electronic interfaces [1] and in the field of health care. It is highly likely that within 10 years, computer chips will be integrated into garments and wearable technology will become as common as mobile phones. One of the most practical techniques to make textiles electrically conductive is to apply conductive polymers to the fabric surface. Conductive polymers have a comparable degradation lifetime to textile materials [2]. The coating is easily applied and does not have a significant effect on the fabric softness. Therefore, conductive polymer coated textiles are promising materials for wearable electronics.

Conductive polymers have attracted the attention of a great number of researchers in the textile field due to their potential applications in composites with natural, artificial or synthetic fibers. The affinity to several kinds of fibers, yarns and fabrics with doped conjugated polymers, permits the production of composite textiles with improved electrical properties. Polypyrrole (Ppyr) is one of the most suitable conductive polymers for deposition on textile materials due to its excellent conductivity and relevant environmental stability [3-5]. Ppyr is commonly produced by electrochemical synthesis or chemical oxidative polymerization in aqueous solution and shows good affinity with natural and artificial fibers. Textile substrates can be easily covered with a Ppyr layer by immersion of the fabric in the polymerization solution containing pyrrole (Pyr), an oxidant and a doping agent [6-8].

In the wake, the interest shown by the microelectronics industry for intrinsic conducting polymers (ICPs) which is from the textile, and paper industries for the wide range of new functionalities of ICPs have increased progressively during the last two decades. Conductive fabrics from natural (wool and cellulose) $[9,10]$ and synthetic [11] fibers have been elaborated by polymerizing 
in situ pyrrole using ferric compounds as oxidizing agents.

Recent research shows that polypyrrole can be embedded in natural and manmade cellulose based fibers, such as cotton, viscose, cupro and lyocell, by means of internal polymerization taking place in the amorphous region of the fibers [12]. Pyrrole was applied by a simple finishing process at room temperature from an aqueous solution of the monomer that penetrates into the cellulose substrate similarly to a non-reactive dye. Subsequent polymerization caused its permanent insolubility in the internal fiber structure, leading to high levels of fastness to washing and to light exposure, with significant modification of the mechanical properties of the fibers [13-15]. The type of monomer and the dopant anion, concentrations, and synthesis variables such as time, temperature, agitation, and sequence of exposure to chemicals vary among different research groups, and each of these parameters has an effect on the electrical, morphological and mechanical properties, and the stability of the resulting material [14].

In this work, the chemical oxidation polymerization process reaction conditions (monomer concentration, catalyst: dopant ratio and temperature) have been optimized in order to obtain the best properties of the treated fabric. We report the effects of coating cotton fabric by conductive polypyrrole films on electrical properties, color strength, weight gain, tensile strength and elongation.

\section{Experimental and Methods}

\subsection{Fabrics and Materials}

White cotton fabric was obtained from INOTEX Company, Czech Republic, Pyrrole monomer from Merck. Ferric chloride of analytical reagent grade from BDH Chemicals was used. Tetra-ethylammonium p-Toluenesulfonate $\left[\left(\mathrm{C}_{2} \mathrm{H}_{5}\right)_{4} \mathrm{~N}\left(\mathrm{CH}_{3} \mathrm{C}_{6} \mathrm{H}_{4} \mathrm{SO}_{3}\right)\right]$ was obtained from Aldrich Chemicals. All other chemicals used were of analytical grade. All aqueous solutions were prepared with distilled water.

\subsection{Methods}

\subsubsection{Preparation of Polypyrrole Coated Fabric}

Preparation of polypyrole coated fabric obtained by two steps as follows:

1) A piece of fabric was soaked in an aqueous solution containing different amounts of both an oxidant (Ferric Chloride) and dopant (TEAp-TS) for about 20 minutes with fixed ratio between them $[2: 1(\mathrm{M} / \mathrm{M})]$ and a fabric/ bath ratio of 1:25, then the substrate was pressed with an automatic padder with considering in mind that the wet pick-up (the weight of the liquid as the percentage weight of the substrates) is about $100 \%$.

2) The treated sample with catalyst and dopant was allowed to soak in the Ahiba Nuance dye pots contain a solution of pyrrole in water, the pots were placed in a rapid rotary dyer. The polymerization was carried out for 4 hours at $25^{\circ} \mathrm{C}$ with a fabric/bath ratio of $1: 25$ using different monomer concentrations ( $0.1 \mathrm{M}$ to $0.4 \mathrm{M})$. Agitation was supplied via rotation of the pots at 25 revolutions per minute. Rotation direction was changed every 1 minute. After polymerization the samples were rinsed with water to remove the polymer excess and the samples were dried at ambient conditions. The fabric was black in color resembling polypyrrole.

\subsection{Testing and Analysis}

\subsubsection{Weight Gain Percent}

The amount of PPy deposited on the cotton fabrics was determined by weighing the cotton samples before and after treatment under standard conditions of temperature $\left(20^{\circ} \mathrm{C}\right)$ and relative humidity $(65 \%)$. The percentage weight increase $(\mathrm{W} \%)$ was calculated as follows:

$$
\mathrm{W} \%=\frac{\mathrm{Wf}-\mathrm{Wi}}{\mathrm{Wi}} \times 100
$$

where Wi and Wf are the initial and final weight, respectively.

\subsubsection{Characterization of Electrical Properties}

The conductivity of the PPy-coated cotton fabric was measured using the AATCC 76 two point-probe technique using FLUKA digital multimeter. The bulk resistances of the dried and as prepared samples were measured at room temperature using Professional Digital Multimeter (uni-T, UT70C). The sample conductivity, $\sigma$, was obtained from the measured resistance $R$ according to $\sigma=(1 / R)$.

\subsubsection{Tensile Strength}

Fabric tensile strength test was conducted according to ASTM method 1682 (1994) (using Testometric (M350$5 \mathrm{CT}$ ), which is a standard method for breaking force and elongation of tensile fabrics [16]. The width and the length of the fabric strip were $50 \mathrm{~mm}$ and $200 \mathrm{~mm}$ respectively.

\subsubsection{Color Measurement}

The color intensity expressed as K/S value, of the stained samples before and after treatment was, as a function for polymerization efficiency, determined spectrophotometrically using Datacolor spectrophotometer. K/S was calculated by applying the Kubelka-Munk equation [17].

All the determinations in this work were done in triplicate and the results present mean values.

\subsubsection{Scanning Electron Microscopy (SEM)}

The surface morphology of untreated and treated fabric 
was investigated by using SEM, VEGA (TESCAN, Czech Republic). Before examination, the fabric surface was prepared on an appropriate disk and coated randomly by a spray of gold.

\subsubsection{FT-IR Spectroscopy}

The FT-IR spectra of cotton fabrics treated with polypyrrole were recorded on a FT-IR spectrometer PerkinElmer, in the spectra range $4000-400 \mathrm{~cm}^{-1}$ using the $\mathrm{KBr}$ disc technique. All the measurements in this work were carried out in Faculty of Textile Engineering, Liberec, CZ.

\section{Results and Discussion}

\subsection{Tentative Mechanism of the Polymerization Reaction}

The reaction is initiated by the oxidation of monomer into radical cations, which combine to form dimers. Continuation of the process leads to formation of insoluble oligomers in solution, which deposit on the surface and interstices of the textile fibers and fabrics. Due to low oxidation potential of pyrrole, a wide range of oxidizing agents can be used to initiate polymerization. Oxidant salts such as ferric chloride $\left(\mathrm{FeCl}_{3}\right)$ function as both the oxidant and the dopant agent, and hence the polymer is obtained in the conducting form. Other transition metal salts can also be used as oxidizing agents for the polymerization of pyrrole. The transition metal ion is an electron acceptor. Therefore it oxidizes the $\pi$-electron system of the pyrrole ring at the initiation step. The intermediate steps of the polymerization of pyrrole initiated by $\mathrm{FeCl}_{3}$ are as follows: The pyrrole molecule is oxidized to yield a radical cation as follows [14]:

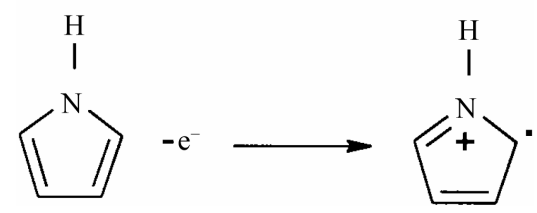

Radical cations recombine to form dications:

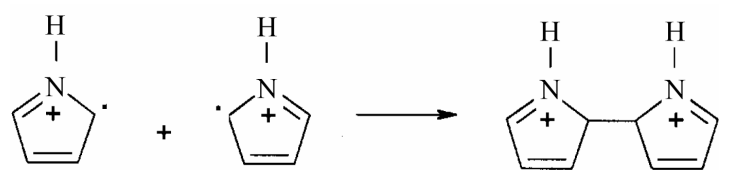

Deprotonation of dication to yield a dimer:
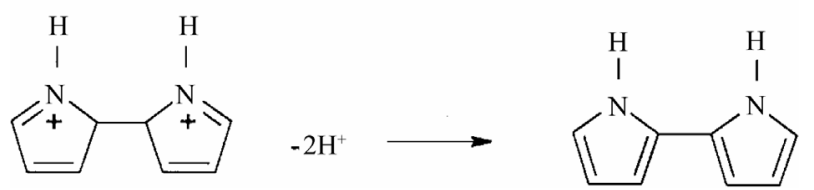

The above process continues by oxidation of the dimer to yield a radical cation of the dimer and the combination of radical cations to form trimers, tetramers, and polymers. Sulfonate anion also attaches itself to pyrrole molecule during chemical polymerization. Oxidation of polypyrrole results in a partial positive charge on the pyrrole ring, which attracts anionic species such as p-toluene sulfonate in order to achieve charge neutrality as follows [14]:

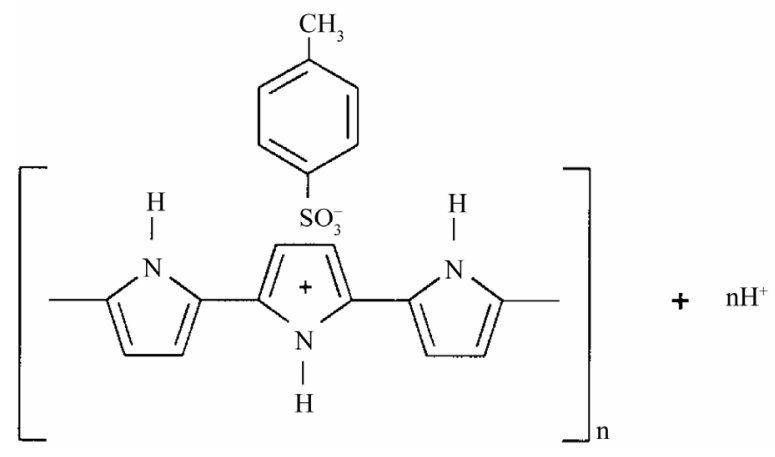

\subsection{Influence of the Ratio between $\mathrm{FeCl}_{3}$ and TEAp-TS}

The effect of $\mathrm{FeCl}_{3}$ concentration in admixtures with TEAp-TS on the fabric properties, expressed as weight gain, resistivity, color strength, tensile strength and antimicrobial properties when the treatment was carried out with pyrrole $(0.2 \mathrm{M} / \mathrm{L})$ using $\mathrm{FeCl}_{3}$ as an oxidant and TEAp-TS as a dopant, which in turn caused a reduction in the moisture regain of the substrate, at $25^{\circ} \mathrm{C}$ for 4 hours are shown in Figures 1-5. It can be seen (Figure 1) that, the use of $\mathrm{FeCl}_{3} / \mathrm{TEAp}$-TS mixture causes increasing of fabric properties. When the ratio between $\mathrm{FeCl}_{3}$ and TEAp-TS in the mixture increases, weight gain increases. This is due to the increase of radical cations number which combined with each others to form polymer, this is arises from $\mathrm{FeCl}_{3}$ acted as oxidizing agent of pyrrole for the polymerization of pyrrole on the fabric surface in presence of TEAp-TS as a doping agent and hence quantity of the polymer on the fabric increased.

Constant parameters: [pyrrole]: $0.2 \mathrm{M} / \mathrm{L}$; polymerization time: $4 \mathrm{~h}$; polymerization temperature: $25^{\circ} \mathrm{C}$.

Figures $\mathbf{2}$ and $\mathbf{3}$ depict that, tensile strength and elongation of the treated fabric increasing by increasing the ratio between $\mathrm{FeCl}_{3}$ and TEAp-TS in the mixture. The increase in tensile strength and elongation of the coated fabric with polypyrrole was attributed to the reinforcing effect of the high strength and modulus conductive polymer coating on the fiber.

Constant parameters: [pyrrole]: $0.2 \mathrm{M} / \mathrm{L}$; polymerization time: $4 \mathrm{~h}$; polymerization temperature: $25^{\circ} \mathrm{C}$.

Constant parameters: [pyrrole]: $0.2 \mathrm{M} / \mathrm{L}$; polymerization time: $4 \mathrm{~h}$; polymerization temperature: $25^{\circ} \mathrm{C}$. 


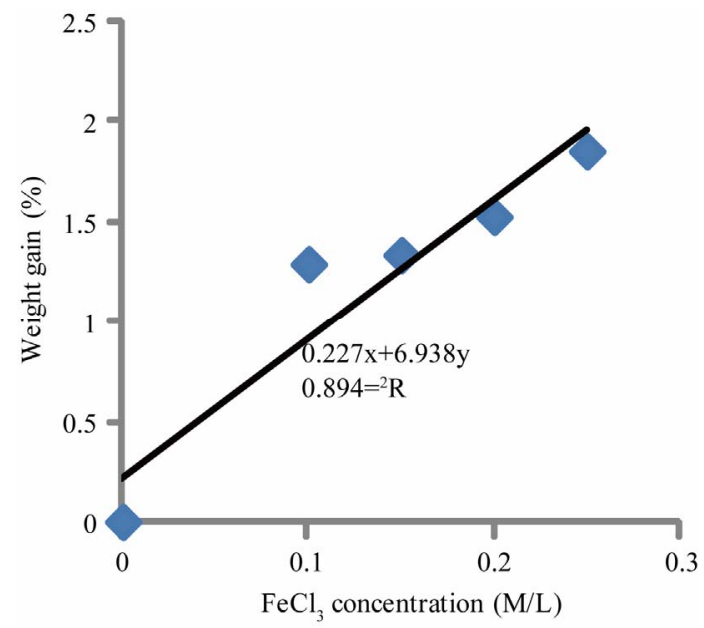

Figure 1. Percent weight gain of treated cotton fabrics with the increase in $\mathrm{FeCl}_{3}$ concentration in the impregnation aqueous solution.

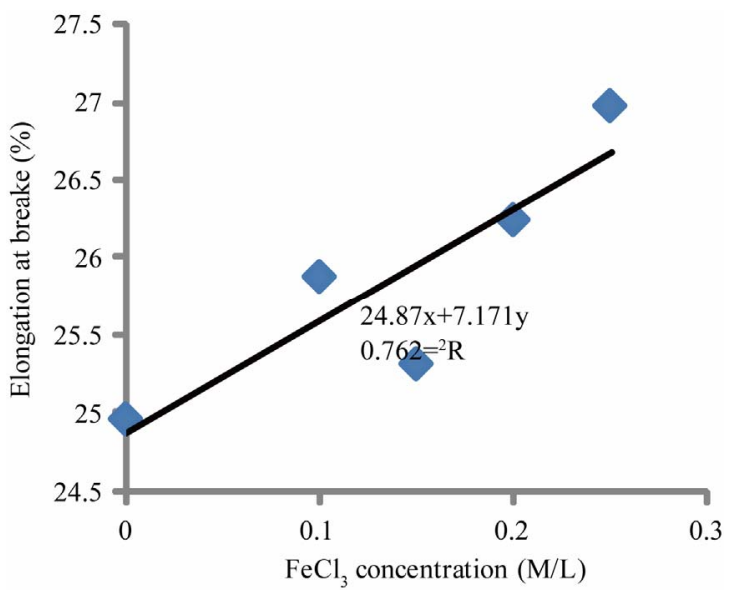

Figure 2. Coated fabric elongation change under $\mathrm{FeCl}_{3}$ concentration effect.

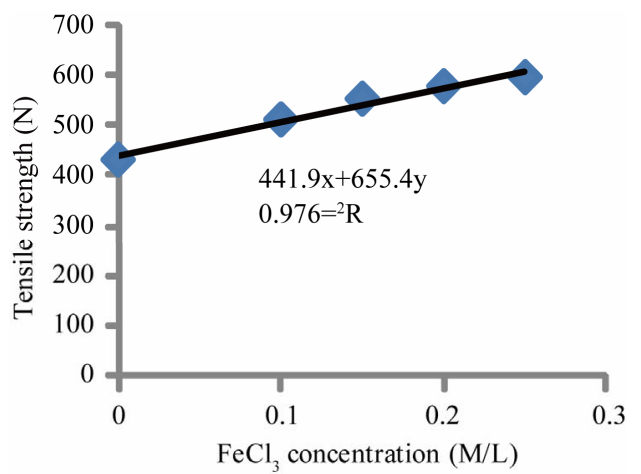

Figure 3. Effect of $\mathrm{FeCl}_{3}$ concentration in the impregnation aqueous solution on the strength of treated fabrics.

Polymerization of Pyrrole resulted in black cotton fabrics, meaning that the polymer completely coated the surface of the fibers. When we measured the depth of color $(\mathrm{K} / \mathrm{S})$ of the coated fabrics we have seen an increase with

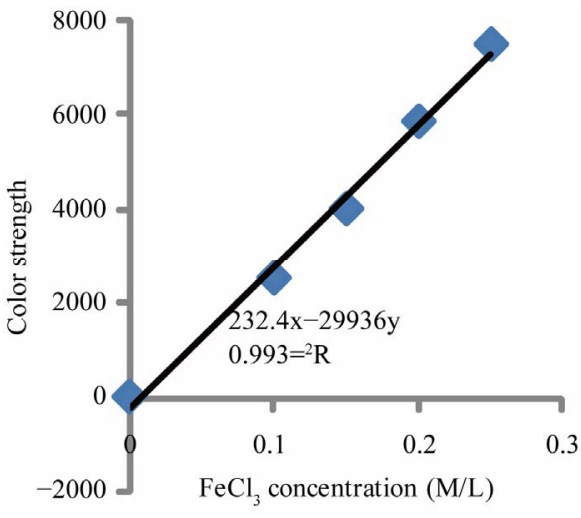

Figure 4. Effect of $\mathrm{FeCl}_{3}$ concentration in the impregnation aqueous solution on the color strength of treated fabrics.

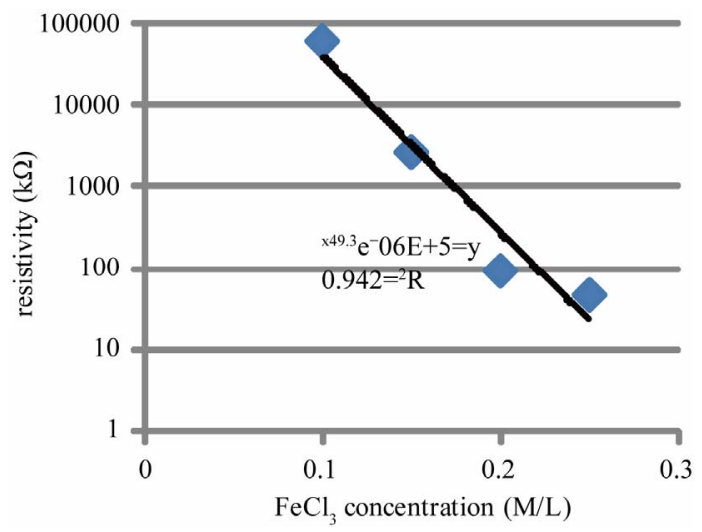

Figure 5. Trend of resistivity of the coated fabric as a function of $\mathrm{FeCl}_{3}$ concentration in the impregnation aqueous solution.

the increase in the ratio between $\mathrm{FeCl}_{3}$ and TEAp-TS in the mixture. Kinetics of depth of color increase is shown in Figure 4.

Constant parameters: [pyrrole]: $0.2 \mathrm{M} / \mathrm{L}$; polymerization time: $4 \mathrm{~h}$; polymerization temperature: $25^{\circ} \mathrm{C}$.

On the other hand, there was a significant decrease in the resistivity of the coated fabric with polypyrrole by increasing the ratio between $\mathrm{FeCl}_{3}$ and TEAp-TS in the mixture. The decrease in the resistivity is arises from conductive polymer layer formation on the fabric surface and increase the amount of polymer on the fabric surface as shown in Figure 5. Current results call for a pad bath formulation consisting of $\mathrm{FeCl}_{3}(0.25 \mathrm{M} / \mathrm{L})$ and TEApTS $(0.125 \mathrm{M} / \mathrm{L})$ as the most appropriate formulation for effecting polymerizing of pyrrole and anchoring on cotton fabrics.

Constant parameters: [pyrrole]: $0.2 \mathrm{M} / \mathrm{L}$; polymerization time: $4 \mathrm{~h}$; polymerization temperature: $25^{\circ} \mathrm{C}$.

\subsection{Influence of the Pyrrole Concentration}

Table 1 and Figure 6 show that the effect of the pyrrole concentration on the coated fabric with polypyrrole 
Table 1. Effect of monomer conc. on some properties of coated fabric with pyrrole.

\begin{tabular}{ccccc}
\hline $\begin{array}{c}\text { Pyrrole Con. } \\
(\mathrm{M} / \mathrm{L})\end{array}$ & $\begin{array}{c}\text { Weight } \\
\text { gain }(\%)\end{array}$ & $\begin{array}{c}\text { Tensile } \\
\text { strength (N) }\end{array}$ & $\begin{array}{c}\text { Elongation } \\
\text { at break (\%) }\end{array}$ & $\begin{array}{c}\text { Color } \\
\text { strength, K/S }\end{array}$ \\
\hline 0 & 0 & 433.9 & 24.97 & 6.3 \\
0.1 & 1.871 & 594.7 & 26.64 & 7450 \\
0.2 & 1.85 & 597.1 & 27.98 & 9246 \\
0.3 & 1.955 & 599.1 & 28.94 & 9565 \\
0.4 & 1.989 & 600.1 & 29.01 & 9579 \\
\hline
\end{tabular}

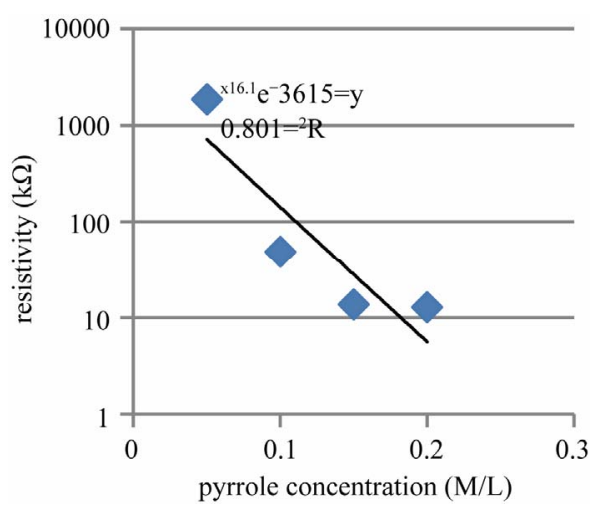

Figure 6. Dependence of the resistivity of the treated fabric on pyrrole concentration in the impregnation aqueous solution.

properties. The cotton fabric treated with $\mathrm{FeCl}_{3}(0.25$ $\mathrm{M} / \mathrm{L})$ and TEAp-TS $(0.125 \mathrm{M} / \mathrm{L})$, the polymerization carried out at $25^{\circ} \mathrm{C}$ for 4 hour with a fabric/ water ratio of $1: 25$ using different monomer concentrations $(0.1 \mathrm{M}$ to $0.4 \mathrm{M}$ ). The amount of polymer was evaluated by measuring the weight increase of fabric samples as a function of pyrrole concentration in solution. We noticed that, by increasing of pyrrole concentration weight gain of the coated fabrics increases up to $0.3 \mathrm{M} / \mathrm{L}$. Above this value no significant increase in the weight gain as shown in Table 1.

Table 1 discloses also that, tensile strength and elongation of the coated fabric with polypyrrole improved by increasing pyrrole concentration. This behavior is attributable to the increased amount of polypyrrole in the composites, in good agreement with the weight increase results shown in Table 1. The same holds true obtained with color strength. During the chemical polymerization, both bulk polymerization (polymers form insoluble precipitations) and polymer deposition on the surface of the substrates occurred. At higher concentration of pyrrole, a better yield of conductive polymer and a better K/S were achieved as displayed in Table 1.

Constant parameters: $\mathrm{FeCl}_{3}$ /TEAp-TS: $0.25 \mathrm{M} / \mathrm{L} /$ $0.125 \mathrm{M} / \mathrm{L}$; polymerization time: $4 \mathrm{~h}$; polymerization temperature: $25^{\circ} \mathrm{C}$.

The trend of resistivity of polypyrrole-coated cotton fabrics as a function of Pyrrole concentration is shown in
Figure 6. Resistivity decreased exponentially with the increasing of monomer concentration in the polymerization bath. In other words, increasing the coating thickness decreased the resistivity. Increasing the pyrrole concentration not only increased the conductivity of the polymer but also increased the polymer film thickness as the bulky anion (dopant) attached itself to the partial positive charge on the oxidized pyrrole ring. This observation points out the key role played by the amount of polypyrrole loaded onto the cotton fabric in determining the ultimate electrical properties of polypyrrole-coated cotton fabrics. These results show that the optimum condition is a Pyrrole concentration of $0.3 \mathrm{M} / \mathrm{L}$ in the polymerization bath. Under these process conditions, the fabric resistivity looks suitable for employment as conductive fabric for application based on EMI Shielding and static charges dissipation.

Constant parameters: $\mathrm{FeCl}_{3}$ /TEAp-TS: $0.25 \mathrm{M} / \mathrm{L} /$ $0.125 \mathrm{M} / \mathrm{L}$; polymerization time: $4 \mathrm{~h}$; polymerization temperature: $25^{\circ} \mathrm{C}$.

\subsection{IR Analysis}

The IR spectrum of the treated cotton fabric, as shown in Figure 7 reveals the presence of a broad band at 3300 $3500 \mathrm{~cm}^{-1}$ attributable to the $\mathrm{OH}$ groups, a strong absorption band at about $1566 \mathrm{~cm}^{-1}$ which is assigned to the $\mathrm{C}=\mathrm{N}$ and $\mathrm{C}=\mathrm{C}$ groups, with notice that this absorption band disappeared in case untreated cotton fabric. These IR spectrums confirm the presence of polypyrrole in the treated cotton fabric. That is, polypyrrole polymerized or deposited on the surface of cotton fabric.

The surface examination of the polypyrrole coated fabrics by SEM (Figures 8(a) and (b)) reveals the deposition of the polypyrrole on the cotton fabric surface; this led to the success of the idea of our paper.

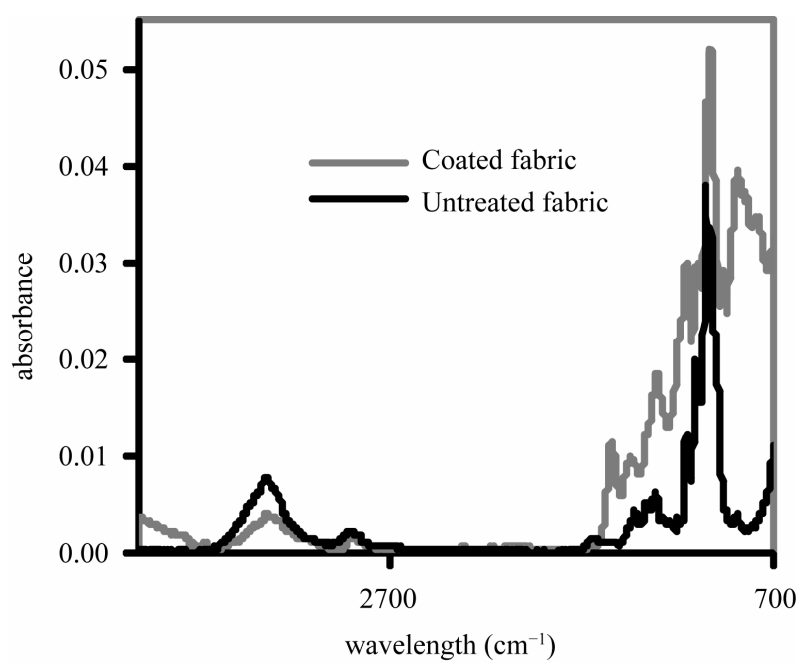

Figure 7. FT-IR spectra, of coated sample with polypyrrole and untreated sample. 


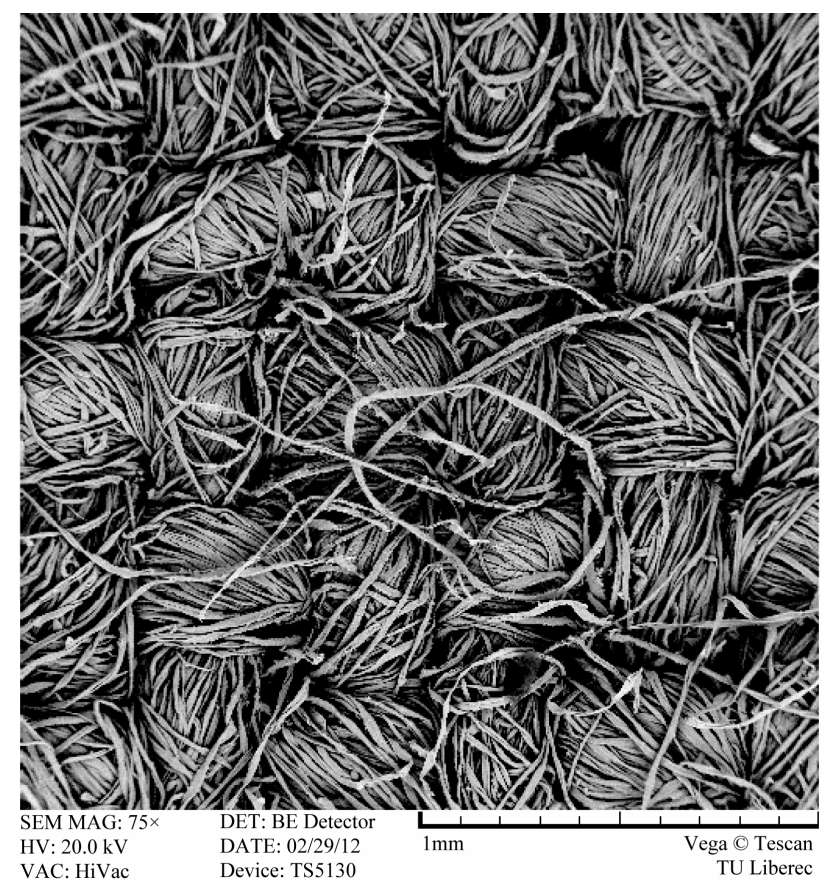

(a)

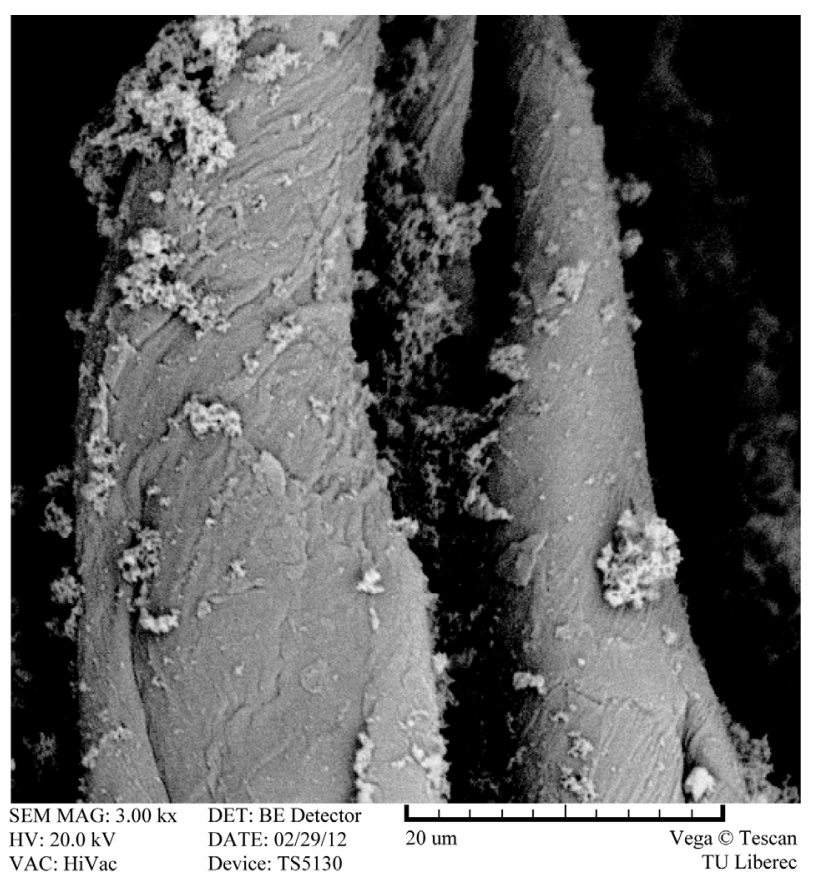

(b)

Figure 8. (a), (b): SEM micrographs showing the deposition of polypyrrole on cotton fabrics.

\section{Conclusion}

Preparation of conductive cotton fabrics, by polymerization of pyrrole in presence of $\mathrm{FeCl}_{3}$ as an oxidizing agent and TEAp-TS as a doping agent, and by the mentioned method in the experiment, resulted in improving in electrical conductivity, weight gain, color strength, tensile strength and elongation of the coated fabrics with polypyrrole. As a result of chemical polymerization, a layer of conductive polymer was deposited on the fabric surface, which affected the overall properties, particularly, electrical conductivity property. The amount of coating polymer in turn causes a reducing resistivity of the coated fabrics. The results reported in this study may open new perspectives for future application of polypyrrole-coated cotton fabrics not only in clothing industry but also in the field of technical textiles.

\section{REFERENCES}

[1] D. De Rossi, A. D. Santa and A. Mazzoldi, "Dressware: Wearable Hardware," Materials Science and Engineering: C, Vol. 7, No. 1, 1999, pp. 31-35. http://dx.doi.org/10.1016/S0928-4931(98)00069-1

[2] J. Wu, D. Zhou, C. O. Too and G. G. Wallace, "Conducting Polymer Coated Lycra," Synthetic Metals, Vol. 155, No. 3, 2005, pp. 698-710.

[3] M. S. Kim, H. K. Kim, S. W. Byun, S. H. Jeong, Y. K. Hong, J. S. Joo, K. T. Song, J. K. Kim, C. J. Lee and J. Y. Lee, "PET Fabric/Polypyrrole Composite with High Electrical Conductivity for EMI Shielding," Synthetic Metals, Vol. 126, No. 2-3, 2002, pp. 233-237. http://dx.doi.org/10.1016/S0379-6779(01)00562-8

[4] A. Varesano, L. Dall'Acqua and C. Tonin, "Electrically Conductive Textiles by in Situ Polymerization of Conjugated Polymers," Polymer Degradation and Stability, Vol. 89, No. 1, 2005, pp. 125-139.

http://dx.doi.org/10.1016/j.polymdegradstab.2005.01.008

[5] B. Huang, G. J. Kang and Y. Ni, "Preparation of Conductive Paper by in-Situ Polymerization of Pyrrole in a Pulp Fibre System," Pulp \& Paper Canada, Vol. 107, No. 2, 2006, pp. 38-41.

[6] I. Cucchi, A. Boschi, C. Arosio, F. Bertini, G. Freddi and M. Catellani, "Bio-Based Conductive Composites: Preparation and Properties of Polypyrrole(PPy)-Coated Silk Fabrics," Synthetic Metals, Vol. 159, No. 3-4, 2009, pp. 246-253.

http://dx.doi.org/10.1016/j.synthmet.2008.09.012

[7] K. S. Jang, H. Lee and B. Moon, "Synthesis and Characterization of Water Soluble Polypyrrole Doped with Functional Dopants," Synthetic Metals, Vol. 143, No. 3, 2004, pp. 289-294.

http://dx.doi.org/10.1016/j.synthmet.2003.12.013

[8] M. K. Song, Y.-T. Kim, B.-S. Kim, J. Kim, K. Char and H. W. Rhee, "Synthesis and Characterization of Soluble Polypyrrole Doped with Alkylbenzenesulfonic Acids," Synthetic Metals, Vol. 141, No. 3, 2004, pp. 315-319. http://dx.doi.org/10.1016/j.synthmet.2003.07.015

[9] L. Dall'Acqua, C. Tonin, R. Peila, F. Ferrero and M. Catellani, "Vapour Phase Polymerisation of Pyrrole on Cellulose-Based Textile Substrates," Synthetic Metals, Vol. 156, No. 5-6, 2006, pp. 379-386. http://dx.doi.org/10.1016/j.synthmet.2005.12.021

[10] A. Varesano, L. Dall'Acqua and C. Tonin, "A Study on the Electrical Conductivity Decay of Polypyrrole Coated 
Wool Textiles," Polymer Degradation and Stability, Vol. 89, No. 1, 2005, pp. 125-132.

http://dx.doi.org/10.1016/j.polymdegradstab.2005.01.008

[11] T. Lin, L. Wang, X. Wang and A. Kayan, "Polymerising Pyrrole on Polyester Textiles and Controlling the Conductivity through Coating Thickness," Thin Solid Films, Vol. 479, No. 1-2, 2005, pp. 77-82. http://dx.doi.org/10.1016/j.tsf.2004.11.146

[12] L. Dall'Acqua, C. Tonin, R. Peila, F. Ferrero and M. Catellani, "Performances and Properties of Intrinsic Conductive Cellulose-Polypyrrole Textiles," Synthetic Metals, Vol. 146, No. 2, 2004, pp. 213-221. http://dx.doi.org/10.1016/j.synthmet.2004.07.005

[13] F. Y. Li, S. Y. Yan and X. W. Cheng, "Performance of Electrically Conductive Fabrics Based on Polyester/ Metal Wire Wrapped Yarns," Journal Advance Materials Research, Vol. 28, No. 7, 2011, pp. 2543-2546. http://dx.doi.org/10.4028/www.scientific.net/AMR.287-2
90.2543

[14] A. Kaynak, L. J. Wang, C. Hurren and X. G. Wang, "Characterization of Conductive Polypyrrole Coated Wool Yarns," Fibers and Polymers, Vol. 3, No. 1, 2002, pp. 24-30. http://dx.doi.org/10.1007/BF02875365

[15] L. J. Wang, T. Lin, X. G. Wang and A. Kaynak, "Frictional and Tensile Properties of Conducting Polymer Coated Wool and Alpaca Fibers," Fibers and Polymers, Vol. 6, No. 3, 2005, pp. 259-262. http://dx.doi.org/10.1007/BF02875651

[16] American Society for Testing and Materials (ASTM), "Standard Test Method," Vol. 1682, ASTM, West Conshohocken, 1994.

[17] B. D. Judd and G. Wysezecki, "Colour in Business Science and Industry," 3rd Edition, John Wiley and Sons, New York, 1975. 